\title{
Temperamento de bajo miedo y respuesta cardiaca de defensa
}

\author{
Carlos Ventura Bort \\ cventura@uji.es \\ RAÚL LÓPEZ PENAdÉS \\ penades@uji.es \\ Àngels EstelLER CANO \\ aestelle@uji.es \\ ROSARIO POY GIL \\ poy@uji.es \\ Pilar Segarra Cabedo \\ segarra@uji.es \\ ALICIA FONFRÍA MORENO \\ afonfria@uji.es \\ PABLO RIBES GUARDIOLA \\ pribes@uji.es \\ JAVIER MOLTÓ BROTONS ${ }^{1}$ \\ molto@uji.es
}

\section{Resumen}

La psicopatía se caracteriza por la presencia de un temperamento de bajo miedo asociado con una menor o deficitaria activación del sistema motivacional defensivo. Uno de los índices psicofisiológicos de reactividad defensiva más consolidados es la respuesta cardiaca de defensa (RCD). La RCD es un patrón complejo de cambios en la frecuencia cardiaca compuesto por componentes acelerativos y desacelerativos producidos por la presentación de una estimulación auditiva intensa e inesperada; su segundo componente acelerativo (A2) se considera un índice del nivel de activación del sistema motivacional defensivo. Estudios previos encontraron que el factor de 'Dominancia/Ausencia de Miedo' del Psychopathic Personality Inventory-Revised (PPI-R-I; PPI-R; Lilienfeld \& Widows, 2005) -que evalúa los rasgos relacionados con el temperamento de bajo miedo de la psicopatía- se asociaba con una disminución en el componente A2 de la RCD (cf. Esteller et al., 2010). El presente estudio pretendió investigar la contribución diferencial de las subescalas del PPI-R-I ('Influencia Social', 'Inmunidad

1. Esta investigación ha sido posible gracias a la financiación de los proyectos SEJ2007-61742 del Ministerio de CIEncia e Innovación y PSI2011-22559 del Ministerio de Economía y Competitividad. Correspondencia: Javier Moltó, Laboratorio de Neurociencia Afectiva, Departamento de Psicología Básica, Clínica y Psicobiología, Universitat Jaume I, Avenida Sos Baynat s/n, 12071, Castellón, España. E-mail: molto@uji.es. 
al Estrés' y 'Ausencia de Miedo') en la reducción del componente A2 de la RCD, utilizando una muestra de 50 estudiantes mujeres. Los resultados mostraron que las puntuaciones en la subescala de 'Ausencia de Miedo' predecían una menor aceleración cardiaca al inicio del componente $\mathrm{A} 2(8 \%$ de la varianza, $B=-.30, p<.05)$. Este resultado sugiere que son exclusivamente las características de falta de miedo $-y$ no otras características afectivo/interpersonales de la psicopatía- las responsables de la menor reactividad cardiaca defensiva, sumándose, así, a la evidencia empírica que relaciona el temperamento de bajo miedo que subyace a la psicopatía con el déficit en la activación del sistema motivacional defensivo.

Palabras clave: psicopatía, temperamento de bajo miedo, ausencia de miedo, respuesta cardiaca de defensa.

\section{Abstract}

Psychopathy is featured by a low fear temperament related to a reduced, or even deficient, activation of the defensive motivational system. The cardiac defense response (CDR) has been one of the most used psychophysiological indexes to study defensive reactivity. CDR is characterized by a complex pattern of heart rate changes with accelerative and decelerative components produced by an intense, unexpected auditory stimulus. The second accelerative component of CDR has been proposed as an index of the defensive motivational system activation level. Prior studies (Esteller et al., 2010) observed that the 'Fearless Dominance' factor of the Psychopathic Personality Inventory-Revised (PPI-R-I; PPI-R; Lilienfeld \& Widows, 2005), which assesses the low fear tendencies of psychopathy, predicted a reduced cardiac acceleration of the A2 component. The present study examined the differential contribution of the PPI-R-Isubscales ('Social Potency', 'Stress Immunity', and 'Fearlessness') on the reduced A2 acceleration component. Results showed that only fearlessness scores predicted a diminished acceleration at the beginning of the $\mathrm{A} 2$ component ( $8 \%$ of variance, $B=-.30, p<.05$ ). These results suggest that the reduced cardiac defensive reactivity is specifically related to low dispositional fear, and not to other affective/interpersonal traits of psychopathy, adding to a growing body of data indicating the association between the low fear tendencies of psychopathy and the diminished activation of the defensive motivational system.

Keywords: Psychopathy, low fear temperament, Fearlessness, Cardiac Defense Response.

\section{Introducción}

\section{La psicopatía}

La psicopatía es un trastorno de la personalidad que se caracteriza por la presencia de rasgos afectivo/interpersonales como el desapego emocional, la crueldad, la grandiosidad o la falta empatía, así como por la existencia de características de desviación social entre las que 
se incluyen el pobre control conductual, la impulsividad y la irresponsabilidad (Cleckley, 1941/1976; Hare, 1991, 2003). Una de las propuestas explicativas del trastorno más consolidadas es la hipótesis de una ausencia de miedo (Lykken, 1957). Este enfoque teórico basado en la evidencia experimental postula que la característica central de la psicopatía es la presencia de déficits emocionales ante la estimulación aversiva (Fowles, 1980; Hare, 1965; Hare y Quinn, 1971; Lykken, 1957).

Lykken (1957) observó que los psicópatas mostraban una adquisición deficitaria de las respuestas condicionadas de miedo, así como una extinción más rápida. Diversos estudios han replicado estos pioneros resultados (Birbaumer et al., 2005; Flor, Birbaumer, Hermann, Ziegler y Patrick, 2002), poniendo de manifiesto que los psicópatas presentan un déficit en el nivel afectivo-evaluativo del aprendizaje (i. e., los psicópatas no generan la esperada asociación emocional entre el estímulo condicionado y el estímulo incondicionado), pero no en el nivel cognitivo (i. e., los psicópatas evalúan el estímulo nocivo de manera similar a los no psicópatas). Asimismo, los psicópatas tampoco muestran la potenciación normal de la respuesta defensiva de parpadeo ante la estimulación aversiva (Herpertz et al., 2001; Levenston, Patrick, Bradley y Lang, 2000; Pastor, Moltó, Vila y Lang, 2003; Patrick, Bradley y Lang, 1993), lo cual sugiere la existencia en estos sujetos de un déficit en el procesamiento emocional de la estimulación aversiva.

Hallazgos recientes, sin embargo, cuestionan si los déficits emocionales son características exclusivas de la psicopatía -tal y como postula la hipótesis de un bajo miedo- o si, más bien, son debidos específicamente a las características afectivas e interpersonales del trastorno. Esta controversia nace a raíz de la obtención de correlatos diferenciales entre los déficits emocionales en la psicopatía y los componentes afectivo/interpersonales y de desviación social del trastorno, lo que parece apuntar hacia etiologías distintas para ambos componentes: el componente afectivo/interpersonal estaría relacionado con una reducida activación del sistema motivacional defensivo, y el componente de desviación social reflejaría déficits asociados al control ejecutivo (Fowles y Dindo, 2006; Patrick, 2007; Patrick y Bernat, 2009). Esta conceptuación dual de la psicopatía, también denominada modelo dual (Fowles y Dindo, 2006; Patrick, 2007; Patrick y Bernat, 2009), considera el trastorno como la confluencia de dos disposiciones temperamentales independientes de miedo/ausencia de miedo (más marcada en el componente afectivo/interpersonal de la psicopatía) y vulnerabilidad externalizante (más marcada en el componente de desviación social).

Desde este punto de vista, los individuos que posean características de ambas dimensiones mostrarán muchos rasgos subyacentes a cada una (p. ej., resiliencia emocional y social y asertividad, combinadas con imprudencia, y conductas impulsivas), pero algunos individuos pueden exhibir solo características de un único componente. Esta visión tiene implicaciones tanto para la conceptuación criminal, como para la conceptuación no criminal de la psicopatía (Hall y Benning, 2006; Ishikawa, Raine, Lencz, Birhle, y Lacasse, 2001). Así, la presencia de altos niveles en la dimensión de miedo/ausencia de miedo pero de niveles normales en la dimensión de vulnerabilidad externalizante tendría como resultado una manifestación no criminal de los rasgos psicopáticos (Patrick, 2007). La psicopatía criminal y la psicopatía no criminal, por tanto, pueden entenderse como manifestaciones de distinto grado dentro de un mismo continuo, donde la reducida reactividad defensiva que subyace al temperamento de bajo miedo estaría compartida por las manifestaciones criminal y no criminal del trastorno.

En esta línea, la investigación en psicopatía en la actualidad se ha centrado en abordar principalmente dos cuestiones: por una parte, la continuidad etiológica desde la población criminal a la población normal de las diferentes manifestaciones fenotípicas de la psicopatía (Hall y Benning, 2006) y, por otra, la búsqueda de índices y marcadores psicofisiológicos que 
aporten mayor evidencia acerca de las relaciones divergentes de los dos grandes componentes de la psicopatía con distintos déficits conductuales y afectivos (Patrick y Bernat, 2009).

En cuanto a la primera cuestión, recientemente se ha demostrado en población normal el déficit de los psicópatas en la adquisición del miedo condicionado (López, Poy, Patrick y Moltó, 2013) y su reducida potenciación de la respuesta de parpadeo en contextos aversivos (Benning, Patrick y lacono, 2005; Dvorak-Bertsch, Curtin, Rubinstein y Newman, 2009; Vanman, Mejia, Dawson, Schell y Raine, 2003).

Por lo que respecta a la segunda cuestión, las características de desviación conductual de la psicopatía se han asociado con marcadores psicofisiológicos de vulnerabilidad externalizante, como los componentes P300 y ERN de los potenciales evocados (lacono, Malone y McGue, 2003; Hall, Bernat y Patrick, 2007), mientras que solo los rasgos afectivo/interpersonales del trastorno se han visto relacionados con el deficitario aprendizaje emocional (López et al., 2013) y la falta de potenciación del reflejo de sobresalto (Patrick, 1994; Patrick, Bradley y Lang, 1993; Poy et al., 2009; Vaidyanathan et al., 2011). El examen de otros índices psicofisiológicos de la activación defensiva contribuiría sin lugar a dudas a añadir mayor evidencia empírica a una conceptuación dual de la psicopatía. Uno de los índices de activación defensiva más válidos y consolidados es la respuesta cardiaca de defensa.

\section{La respuesta cardiaca de defensa}

La respuesta cardiaca de defensa (RCD; Vila y Fernández, 1989; Vila et al., 2007) es un patrón complejo de cambios en la frecuencia cardiaca que muestra diferentes componentes acelerativos y desacelerativos a lo largo de los 80 segundos posteriores a la presentación de un estímulo intenso, aversivo e inesperado. El patrón se caracteriza por dos componentes acelerativos y desacelerativos en orden secuencial alterno: aceleración-desaceleración-aceleración-desaceleración (Vila y Fernández, 1989). De acuerdo con el modelo atencional-motivacional de la defensa cardiaca (Vila et al., 2007), los dos componentes acelerativos/desacelerativos secuenciales parecen reflejar la sucesión de dos fases defensivas diferentes que representarían la transición de la atención a la acción: una primera fase defensiva atencional dirigida a interrumpir la actividad presente y analizar el peligro potencial, y una segunda fase defensiva motivacional, dirigida a preparar al organismo para la defensa activa. Dentro de esta segunda fase, el componente acelerativo (A2) se ha propuesto como un índice de la activación del sistema motivacional defensivo (p. ej., López, Poy, Pastor, Segarra y Moltó, 2009; Ramírez, Sánchez, Fernández, Lipp y Vila, 2005). Esta propuesta se encuentra respaldada por la evidencia de que el componente A2 aumenta en individuos normales durante la visión de imágenes aversivas (en comparación con la visión de imágenes neutras; Sánchez et al., 2002), y en individuos fóbicos durante la visión de imágenes relacionadas con su fobia (v. imágenes no fóbicas; Ruiz-Padial, Mata, Rodríguez, Fernández y Vila, 2005).

\section{Psicopatía y respuesta cardiaca de defensa}

Un estudio previo de nuestro laboratorio (Esteller et al., 2010) examinó la relación entre los componentes de la psicopatía y las diferencias individuales en la reactividad defensiva, medida a partir de la RCD, en una muestra de estudiantes evaluados mediante el Psychopathic Personality Inventory-Revised (PPI-R; Lilienfeld y Andrews, 1996; Lilienfeld y Widows, 2005; véase el apartado de Método para una descripción más extensa del instrumento). Como era de esperar, los resultados mostraron patrones diferentes de la RCD en función de las puntua- 
ciones en el PPI-R: los participantes con puntuaciones más elevadas mostraron un componente A2 de menor amplitud que los participantes con puntuaciones bajas. Además, estas diferencias relacionadas con la psicopatía en el componente A2 venían explicadas por los rasgos afectivos e interpersonales del trastorno (factor de 'Dominancia/Ausencia de Miedo' del PPI-R), y no por sus rasgos externalizantes.

El trabajo de Esteller et al. (2010) proporciona evidencia sobre la asociación entre el componente afectivo/interpersonal de la psicopatía y la reducida reactividad defensiva -lo que a su vez respalda la continuidad etiológica del componente de bajo miedo de este trastorno entre las poblaciones normal y penitenciaria-, y sugiere al mismo tiempo que el segundo componente acelerativo de la RCD puede ser un correlato psicofisiológico útil para el estudio de los déficits emocionales de la psicopatía.

En esta línea de investigación, el presente trabajo se plantea con objeto de examinar en mayor profundidad cuáles son los rasgos afectivo/interpersonales más relevantes en la explicación del reducido componente A2 de los psicópatas: la falta de ansiedad anticipatoria, tanto social como física (ausencia de miedo), la escasa evitación de daños (inmunidad al estrés), o el nivel elevado de dominancia interpersonal (influencia social). El objetivo del presente trabajo es, concretamente, investigar la contribución diferencial de las distintas subescalas que componen el factor de 'Dominancia/Ausencia de Miedo' del PPI-R -Ausencia de miedo, Inmunidad al Estrés e Influencia Social- en la reducida amplitud del segundo componente acelerativo (A2) de la RCD.

\section{Método}

\section{Participantes}

Las participantes fueron 50 mujeres estudiantes de grado de Psicología de la Universitat Jaume I con edades comprendidas entre los 17 y los 37 años $(x=19.62$; D. T. = 3.15). Ninguna presentaba trastornos cardiovasculares o deficiencias sensoriales ni estaba sometida a tratamiento farmacológico. Todas las participantes recibieron una bonificación de medio punto en su nota final por su intervención en el estudio.

\section{Cuestionario}

El Psychopathic Personality Inventory-Revised (PPI-R; Lilienfeld y Widows, 2005) es una de las medidas de autoinforme más utilizadas en la investigación de la psicopatía en muestras no penitenciarias. Consta de 154 ítems a los que el sujeto responde mediante una escala de elección múltiple de 4 puntos. El PPI-R proporciona una puntuación global de psicopatía y puntuaciones en 8 subescalas -Inmunidad al Estrés, Influencia Social, Ausencia de Miedo, Inconformismo Rebelde, Externalización de la Culpa, Ausencia Despreocupada de Planes, Egocentrismo Maquiavélico y Frialdad Emocional-. Siete de las subescalas, a su vez, se agrupan en dos factores ortogonales de orden superior (Benning, Patrick, Hicks, Blonigen y Krueger, 2003; López, 2010; Ross, Benning, Patrick, Thompson y Thurston, 2009): Dominancia/Ausencia de Miedo o PPI-R-I(las tres primeras) e Impulsividad Egocéntrica o PPI-R-Il (las cuatro siguientes). Así, el factor Dominancia/Ausencia de Miedo agrupa las características afectivo/interpersonales de la psicopatía, y el factor Impulsividad Egocéntrica recoge las características de desviación social del trastorno (Benning, Patrick, Salekin y Leistico, 2005; Lilienfeld y Widows, 2005). La subescala Frialdad Emocional no satura en ninguno de estos dos factores. 
En la tabla 1 se muestran los estadísticos descriptivos de las puntuaciones totales del PPI-R, de los factores y de las subescalas en la muestra del presente trabajo.

Tabla 1

Estadísticos descriptivos (mediana, media, desviación típica y rango) de las puntuaciones totales, en los factores $y$ en las subescalas del PPI-R en la muestra experimental $(N=50)$

\begin{tabular}{lccccc}
\hline & MEDIANA & MEDIA & D. T. & MíN. & MÁX. \\
\hline Inmunidad al Estrés & \multicolumn{1}{c}{31} & 30.4 & 6.26 & 17 & 46 \\
Influencia Social & \multicolumn{1}{c}{46} & 45.8 & 9.1 & 24 & 65 \\
Ausencia de Miedo & \multicolumn{1}{c}{33.5} & 31.72 & 8.81 & 14 & 46 \\
Egocentrismo Maquiavélico & \multicolumn{1}{c}{35} & 36.06 & 9.19 & 20 & 62 \\
Inconformismo Rebelde & 35 & 34.68 & 7.98 & 15 & 50 \\
Externalización de la Culpa & 31 & 31 & 7.92 & 15 & 48 \\
Ausencia Despreocupada de Planes & 34 & 34.8 & 7.55 & 21 & 57 \\
Frialdad Emocional & 26 & 26.8 & 5.7 & 17 & 46 \\
PPI-R-I & 109.5 & 107.98 & 15.38 & 61 & 148 \\
PPI-R-II & 138 & 136.54 & 21.19 & 72 & 184 \\
PPI-R & 274.5 & 271.32 & 31.7 & 186 & 336 \\
\hline
\end{tabular}

Nota: PPI-R-I: Dominancia/Ausencia de Miedo; PPI-R-II: Impulsividad Egocéntrica; PPI-R: Puntuación Total en el Psychopathic Personality Inventory-Revised (Lilienfeld y Widows, 2005).

\section{Tarea experimental}

La tarea de reacción defensiva duraba 10 minutos: 8 minutos de periodo de adaptación, seguidos de 15 segundos de línea base antes de la administración de la estimulación auditiva y de 80 segundos posteriores a la presentación del estímulo. La tasa cardiaca (TC) se registró de forma continua segundo a segundo durante los 15 segundos anteriores y los 80 segundos posteriores a la presentación del estímulo.

Se utilizó como estímulo auditivo un ruido blanco de 500 ms de duración, 110 dB de intensidad y un tiempo de subida virtualmente instantáneo activado automáticamente.

\section{Aparatos y variables fisiológicas}

La presentación del estímulo y la adquisición de la señal bioeléctrica fueron controladas mediante el software VPM 11.8 (cook, 2001). Este software controlaba la tasa de muestreo del registro de la TC, permitiendo la digitalización de los datos y su posterior almacenamiento en un ordenador PC Pentium (compaq V70). 
La TC se registró y amplificó mediante un módulo Coulbourn V77-26, utilizando sensores de tamaño estándar $(8 \mathrm{~mm})$ de $\mathrm{Ag} / \mathrm{AgCl}$ colocados siguiendo la derivación II. La señal se amplificó por 5000 y se utilizaron filtros de paso alto y paso bajo de $13 \mathrm{~Hz}$ y de $40 \mathrm{~Hz}$, respectivamente. La señal fue tratada, corregida y transformada en valores de cambio de tasa cardiaca mediante el software, basado en MatLab, Kardia V1.3 (Perakakis, Joffily, Taylor, Guerra y Vila, 2010). El ruido blanco se presentaba mediante auriculares TDH 49P de Telephonics, y fue calibrado previamente mediante un sonómetro (Brüel y Kjaer modelo 2235), utilizando un oído artificial (Brüel y Kjaer, modelo 4153).

\section{Procedimiento}

Los datos del cuestionario fueron recogidos de manera grupal antes de la sesión experimental, que cada participante realizaba individualmente. Puesto que esta tarea formaba parte de una investigación más extensa, no fue la única tarea realizada en la sesión, aunque sí fue la primera para todas las participantes. A su llegada al laboratorio, a cada participante se le entregaba el consentimiento donde se indicaba la naturaleza del experimento. Tras su aceptación, era informada de que se iban a registrar diferentes variables psicofisiológicas durante unos 10 minutos. Por último, se colocaban los auriculares, se reducía la iluminación de la habitación a un nivel de penumbra establecido previamente y se cerraba la puerta, dando comienzo al experimento. Una vez finalizada la tarea de reacción defensiva, la participante continuaba la sesión experimental que duraba aproximadamente dos horas.

\section{Análisis estadísticos}

Las puntuaciones directas obtenidas se transformaron en puntuaciones de cambio. Para ello, se restó la media de los valores correspondientes a los 15 segundos de línea base a los valores de la TC correspondientes a los 80 segundos post-estímulo. La forma de la respuesta fue analizada, reduciendo las 80 puntuaciones de cambio a 10 valores correspondientes a las medianas de 10 intervalos progresivamente mayores: dos de 3 segundos, dos de 5 segundos, tres de 7 segundos y tres de 13 segundos (Vila et al., 2007). Concretamente, las medianas 5, 6, 7 y 8 -que conforman el componente A2- fueron las variables objetivo del trabajo, y sobre ellas se centraron los análisis estadísticos posteriores.

Se llevó a cabo un modelo lineal general (MLG) de medidas repetidas sobre el cambio de la TC de cada mediana del componente A2 de la RCD (M5, M6, M7 y M8; variables intra-sujeto). Las puntuaciones de las subescalas Inmunidad al Estrés, Ausencia de Miedo e Influencia Social se incluyeron como variables continuas entre-sujetos.

La relación entre el componente A2 y las subescalas del PPI-R-Ise examinó con más detaIle mediante correlaciones bivariadas de Pearson entre las puntuaciones de los cuestionarios y el cambio de la TC en las medianas 5, 6, 7 y 8, y, cuando fue necesario evaluar la capacidad predictiva de alguna de las subescalas en concreto, mediante análisis de regresión lineal jerárquica sobre las medianas del componente A2. 


\section{Resultados}

Tal y como se esperaba, el MLG reveló que las puntuaciones en la subescala Ausencia de Miedo moderaban el cambio de la TC en el componente A2 $F(1,46)=4.051, p=.05, \eta_{p}^{2}=$ .08). Las puntuaciones de las escalas Influencia Social e Inmunidad al Estrés no mostraron un efecto significativo en el cambio de la TC en el componente A2 $(F s<1)$.

Con el objetivo de explorar más profundamente los resultados se llevaron a cabo correlaciones bivariadas de Pearson entre las puntuaciones de las subescalas del PPI-R-Iy el cambio de la TC en cada uno de los componentes. Como se observa en la tabla 2, las puntuaciones de las subescalas Inmunidad al Estrés e Influencia Social no correlacionaban significativamente con ninguna de las medianas que componen el componente A2 de la RCD ( $p s>.05)$. Sin embargo, las puntuaciones en la subescala Ausencia de Miedo se relacionaron negativa y significativamente con la M6 del componente $(r=-.30, p<.05)$, relación que tendía a permanecer en la M7 $(r=-.27, p=.06)$ y en la M8 $(r=-.26, p=.067)$. El posterior análisis de regresión lineal jerárquica sobre la mediana 6 reveló que las puntuaciones en la subescala de Ausencia de Miedo explicaban un porcentaje significativo de la varianza del cambio de la TC en la M6 (8.3 \%) cuando entraron en tercer lugar en el modelo. Ninguna de las otras dos escalas del PPI-R-Icontribuyó de forma independiente a la predicción de la mediana 6 (véase la tabla 3).

Tabla 2

Correlaciones bivariadas entre las subescalas del PPI-R-Iy el cambio de la TC en las medianas del componente $A 2$ de la RCD (M5, M6, M7 Y M8)

\begin{tabular}{ccccc}
\hline & M5 & M6 & M7 & M8 \\
\hline Inmunidad al Estrés & -.056 & -.007 & .092 & .062 \\
Influencia Social & -.041 & -.141 & -.046 & -.169 \\
Ausencia de Miedo & -.163 & $-.300^{*}$ & $-.270^{+}$ & $-.261^{+}$ \\
\hline
\end{tabular}

${ }^{*} p<.05,{ }^{+} p<.07$

Tabla 3

Resumen del modelo de regresión lineal jerárquica de la media de cambio de la TC en la mediana 6

\begin{tabular}{|c|c|c|c|c|c|c|c|}
\hline \multicolumn{2}{|c|}{ Pasos y variables predictoras } & $\Delta R^{2}$ & $F \Delta R^{2}$ & GI1 & $\mathrm{Gl} 2$ & ßs & Valor de $p$ para \\
\hline Paso 1: & Influencia Social & .020 & .970 & 1 & 48 & -.116 & .414 \\
\hline Paso 2: & Inmunidad al Estrés & .000 & .002 & 1 & 47 & .032 & .823 \\
\hline Paso 3: & Ausencia de Miedo & .083 & 4.264 & 1 & 46 & -.290 & $.045^{*}$ \\
\hline
\end{tabular}

${ }^{*} \mathrm{p}<.05$

La figura 1 ayuda a ilustrar los resultados. Las participantes fueron asignadas al grupo de Alta Ausencia de Miedo $(n=25)$ o al grupo de Baja Ausencia de Miedo $(n=25)$ según sus 
puntuaciones en la subescala de Ausencia de Miedo fueran mayores o menores que la mediana (Mediana $=33.5$ ). Los análisis de comparación de medias t de Student entre grupos para cada mediana del componente A2 de la RCD revelaron que, aunque no había diferencia entre ambos grupos en la mediana $5(t<1)$, las participantes del grupo de Alta Ausencia de Miedo mostraron valores menores en las medianas 6,7 y 8 que las participantes del grupo de Baja Ausencia de Miedo ( $p s<.02)$.

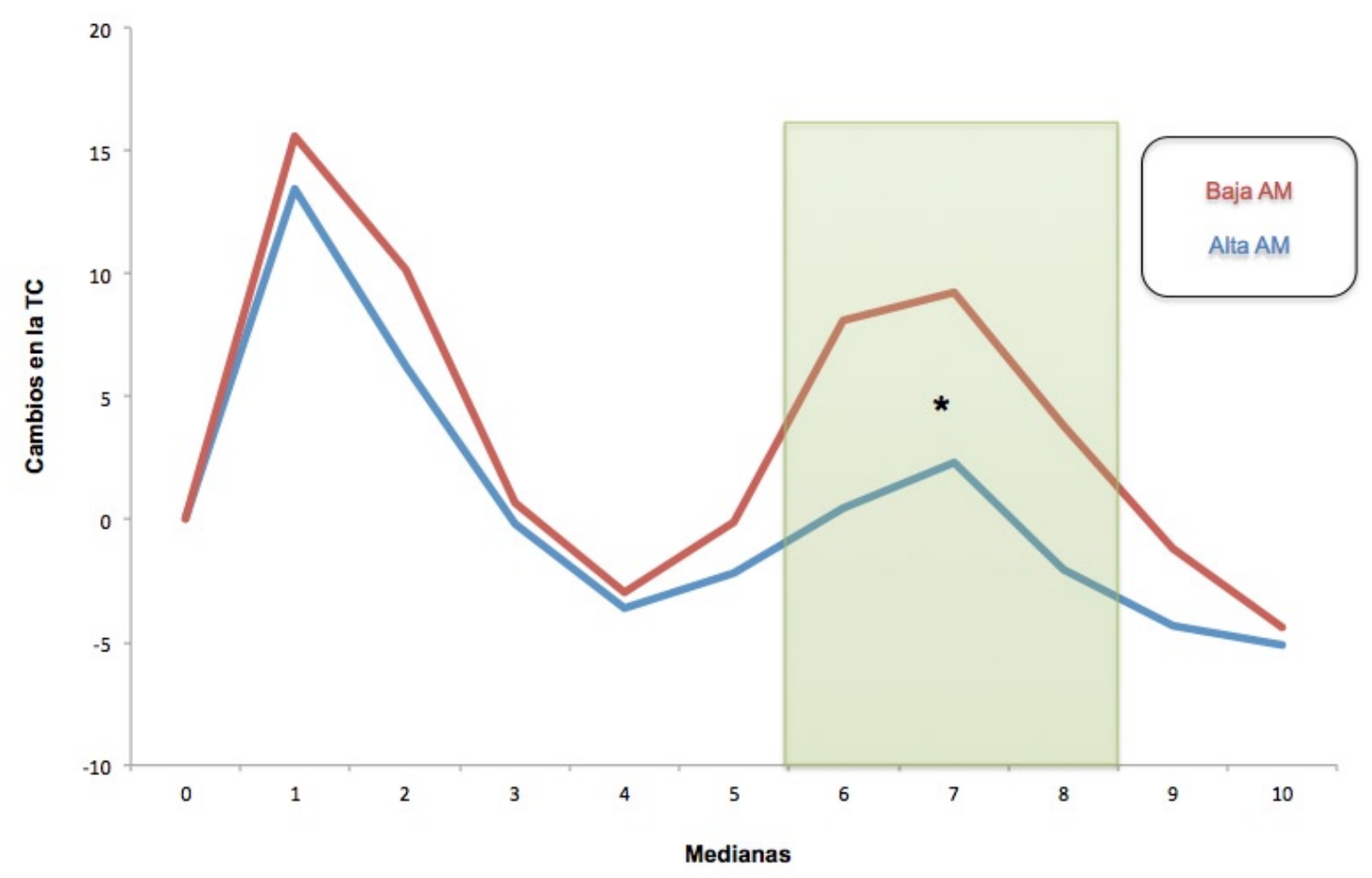

Figura 1. Representación de la RCD de las participantes del grupo de Alta Ausencia de Miedo (Alta AM) y de las participantes del grupo de Baja Ausencia de Miedo (Baja AM)

\section{Discusión y conclusiones}

Este es el primer estudio que evalúa la relación diferencial entre el segundo componente acelerativo de la respuesta cardiaca defensa y las distintas características afectivo/interpersonales de la psicopatía. Los resultados del trabajo mostraron que las puntuaciones de la subescala 'Ausencia de Miedo' del PPI-R predecían la menor amplitud del segundo componente acelerativo de la RCD. Estos hallazgos sugieren que son las características más relacionadas con el temperamento de bajo miedo -tales como la ausencia de ansiedad anticipatoria ante amenazas físicas o la falta de miedo al peligro físico- las que se asocian con una reducida activación del sistema motivacional aversivo/defensivo. En claro contraste, rasgos como la tendencia a ser encantador, la habilidad para influir sobre los demás o la escasa ansiedad social -subescala 'Influencia Social'-, o la capacidad para manejarse con facilidad bajo presión y para mantener la calma ante situaciones estresantes -subescala 'Inmunidad al Estrés'- no parecen encontrarse asociados con un déficit en la activación defensiva. 
Además de sumarse a la evidencia empírica sobre la relación entre el temperamento de bajo miedo de la psicopatía y la deficitaria activación del sistema motivacional defensivo, estos resultados -obtenidos en una muestra de estudiantes universitarias- permiten extender a la psicopatía subclínica las conclusiones de los estudios con psicópatas encarcelados (p. ej., Flor et al., 2002; Pastor et al., 2003; Patrick et al., 1993). Estos resultados, por tanto, constituyen un soporte empírico a la propuesta sobre la existencia de una continuidad etiológica del temperamento de bajo miedo entre las poblaciones criminal y normal (cf. Patrick, 2007; Patrick y Bernat, 2009). Sería conveniente explorar esta cuestión en muestras más amplias y heterogéneas, ya que la homogeneidad de la muestra experimental en nuestro trabajo (mujeres universitarias) limita la capacidad de generalización de las conclusiones en términos de género, edad, nivel educativo, estatus socioeconómico, etc.

Paralelamente, la contribución exclusiva de las características de bajo miedo en la predicción de la reducida activación defensiva aporta datos empíricos a favor de la conceptuación dual de la psicopatía (Fowles y Dindo, 2006; Hall y Benning, 2006; Patrick y Bernat, 2009). De acuerdo con este modelo explicativo, el componente afectivo/interpersonal de la psicopatía difiere etiológicamente del componente de desviación social del trastorno. Concretamente, el primer componente sería la manifestación de una disposición temperamental de ausencia de miedo -asociada, a su vez, a déficits en el sistema motivacional defensivo-, mientras que el segundo componente constituiría la manifestación de una disposición temperamental de vulnerabilidad externalizante, asociada a déficits en los sistemas de control ejecutivo (Fowles y Dindo, 2006; Patrick, 2007).

En suma, este estudio permite concluir que son las características temperamentales de bajo miedo de la psicopatía las que se asocian con una menor activación del sistema motivacional aversivo, apoyando la continuidad etiológica de la psicopatía criminal y subclínica, y respaldando la propuesta de la conceptuación dual de este trastorno de la personalidad como un marco prometedor para el estudio de sus bases etiológicas (cf. López et al., 2013).

\section{Referencias bibliográficas}

Benning, S. D., Patrick, C. J. \& lacono, W. G. (2005). Psychopathy, startle blink modulation, and electrodermal reactivity in twin men. Psychophysiology, 42, 753-762. doi: 10.1111/ j.1469-8986.2005.00353.x.

Benning, S. D., Patrick, C. J., Hicks, B. M., Blonigen, D. M. \& Krueger, R. F. (2003). Factor structure of the Psychopathic Personality Inventory: Validity and implications for clinical assessment. Psychological Assessment, 15, 340-350. doi: 10.1037/1040-3590.15.3.340.

Benning, S. D., Patrick, C. J., Salekin, R. T. \& Leistico, A. R. (2005). Convergent and discriminant validity of psychopathy factors assessed via self-report: A comparison of three instruments. Assessment, 12, 270-289. doi:10.1177/1073191105277110.

Birbaumer, N., Veit, R., Lotze, M., Erb, M., Hermann, C., Grod, W. \& Flor, H. (2005). Deficient fear conditioning in psychopathy: A functional magnetic resonance imaging study. Archives of General Psychiatry, 62, 799-805. doi: 10.1001/archpsyc.62.7.799.

Cleckley, H. (1941/1976). The mask of sanity. St. Louis, MO: Mosby.

Cook, E. W. (2001). VPM reference manual. Birmingham, AL: Author.

Dvorak-Bertsch, J. D., Curtin, J. J., Rubinstein, T. J. \& Newman, J. P. (2009). Psychopathic traits moderate the interaction between cognitive and affective processing. Psychophysiology, 46, 913-921. doi: 10.1111/j.1469-8986.2009.00833.x. 
Esteller, A., Segarra, P., Fonfría, A., Poy, R., López, R., Pastor, M. C., Molés, M. \& Moltó, J. (2010). Cardiac defense response and subclinical psychopathy [Abstract]. Psychophysiology, 47, S41.

Flor, H., Birbaumer, N., Hermann, C., Ziegler, S. \& Patrick, C. J. (2002). Aversive Pavlovian conditioning in psychopaths: Peripheral and central correlates. Psychophysiology, 39, 505-518. doi: $10.1017 / S 004857720239404$.

Fowles, D. C. (1980). The three arousal model: Implications of Gray's two-factor learning theory for heart rate, electrodermal activity, and psychopathy. Psychophysiology, 17, 87-104. doi: 10.1111/j.1469-8986.1980.tb00117.

Fowles, D. C. \& Dindo, L. (2006). A dual-deficit model of psychopathy. En C. J. Patrick (ed.), Handbook of psychopathy (pp. 14-34). New York: Guilford Press.

Hall, J. R. \& Benning, S. D. (2006). The "successful" psychopath: Adaptive and subclinical manifestations of psychopathy in the general population. En C. J. Patrick (ed.), Handbook of psychopathy. New York: Guilford Press.

Hall, J., Bernat, E. M. \& Patrick, C. J. (2007). Externalizing psychopathology and the Error-Related Negativity. Psychological Science, 18 (4), 326-333.

Hare, R. D. (1965). Acquisition and generalization of a conditioned-fear response in psychopathic and non-psychopathic criminals. Journal of Psychology, 59, 367-370.

Hare, R. D. (1991). Hare Psychopathy Checklist-Revised. Toronto, ON: Multi-Health Systems.

Hare, R. D. (2003). Hare Psychopathy Checklist-Revised (2. ${ }^{a}$ ed.). Toronto, ON: Multi-Health Systems.

Hare, R. D. \& Quinn, M. J. (1971). Psychopathy and autonomic conditioning. Journal of Abnormal Psychology, 77, 223-235. doi: 10.1037/ h0031012.

Herpertz, S. C., Werth, U., Lukas, G., Qunaibi, M., Schuerkens, A., Kunert, H. J., Freese, R., Flesch, M., Mueller-Isberner, R., Osterheider, M. \& Sass, H. (2001). Emotion in criminal offenders with psychopathy and borderline personality disorder. Archives of General Psychiatry, 58, 737-745. doi: 10.1001/archpsyc.58.8.737.

lacono, W. G., Malone, S. M. \& McGue, M. (2003). Substance use disorders, externalizing psychopathology, and P300 event-related potential amplitude. International Journal of Psychophysiology, 48, 147-178. doi:10.1016/S0167-8760(03)00052-7.

Ishikawa, S. S., Raine, A., Lencz, T., Bihrle, S. \& Lacasse, L. (2001). Autonomic stress reactivity and executive functions in successful and unsuccessful criminal psychopaths from the community. Journal of Abnormal Psychology, 110, 423-432. doi: 10.1037//0021-843X. 110.3.423.

Levenston, G. K., Patrick, C. J., Bradley, M. M. \& Lang, P. J. (2000). The psychopath as observer: Emotion and attention in picture processing. Journal of Abnormal Psychology, 109, 373-385. doi: 10.1037/0021-843X.109.3.373.

Lilienfeld S. O. \& Andrews, B. P. (1996). Development and preliminary validation of a self-report measure of psychopathic personality traits in noncriminal populations. Journal of Personality Assessment 66, 488-524.

Lilienfeld, S. O. \& Widows, M. R. (2005). Psychopathic Personality Inventory-Revised (PPI-R) professional manual. Odessa, FL: Psychological Assessment Resources.

López, R. (2010). Déficit en el sistema motivacional aversivo en psicópatas subclínicos evaluados mediante el Psychopathic Personality Inventory-Revised (PPI-R). Tesis doctoral. Universitat Jaume I, Castellón, España. Obtenido de:

https://www.educacion.gob.es/teseo/imprimirFicheroTesis.do?fichero=15603.

López, R., Poy, R., Pastor, M. C., Segarra, P. \& Moltó, J. (2009). Cardiac defense response as a predictor of fear learning. International Journal of Psychophysiology, 74, 229-235. doi:10.1016/j. ijpsycho.2009.09.006. 
López, R., Poy, R., Patrick, C. J. \& Moltó, J. (2013). Deficient fear conditioning and self-reported psychopathy: The role of fearless dominance. Psychophysiology, 50, 210-218. doi: 10.1111/ j.1469-8986.2012.01493.x.

Lykken, D. T. (1957). A study of anxiety in the sociopathic personality. Journal of Abnormal and Social Psychology, 55, 6-10. doi: 10.1037/ h0047232.

Pastor, M. C., Moltó, J., Vila, J. \& Lang, P. J. (2003). Startle reflex modulation, affective ratings and autonomic reactivity in incarcerated Spanish psychopaths. Psychophysiology, 40, 934-938. doi: 10.1111/1469-8986.00111.

Patrick, C. J. (1994). Emotion and psychopathy: Startling new insights. Psychophysiology, 31, 319330. doi: 10.1111/j.1469-8986.1994.tb02440.x.

Patrick, C. J. (2007). Getting to the heart of psychopathy. En H. F. Hervé \& Yuille, J. C. (eds.), Psychopathy: Theory, research, and social implications (pp. 207-252). Hillsdale, NJ: Erlbaum.

Patrick, C. J. \& Bernat, E. M. (2009). Neurobiology of psychopathy: A two-process theory. En G. G. Berntson \& Cacioppo, J. T. (eds.), Handbook of neuroscience for the behavioral sciences (pp. 1110-1131). New York, NY: John Wiley \& Sons.

Patrick, C. J., Bradley, M. M. \& Lang, P. J. (1993). Emotion in the criminal psychopath: Startle reflex modulation. Journal of Abnormal Psychology, 102, 82-92. doi: 10.1037/0021 843X.102.1.82.

Perakakis, P., Joffily, M., Taylor, M., Guerra, P. \& Vila, J. (2010). KARDIA: A Matlab software for the analysis of the cardiac interbeat intervals. Computer Methods and Programs in Biomedicine, 98, 83-89. doi:10.1016/j.cmpb.2009.10.002

Poy, R., Segarra, P., Pastor, M. C., López, R., Esteller, A., Fonfría, A., Colomer, C., Tormo, M. P. \& Moltó, J. (2009). Startle modulation and autonomic reactivity in psychopathy: The role of the affective and the antisocial facets [Abstract]. Psychophysiology, 46, S36. doi: 10.1111/j.1469-8986.2009.00920.x

Ramírez, I., Sánchez, M. B., Fernández, M. C., Lipp, O. V. \& Vila, J. (2005). Differentation between protective reflexes: Cardiac defense and startle. Psychophysiology, 42, 732-739. doi: 10.1111/j.1469-8986.2005.00362.x.

Ross, S. R., Benning, S. D., Patrick, C. J., Thompson, A. \& Thurston, A. (2009). Factors of the Psychopathic Personality Inventory: Criterion-related validity and relationship to the BIS/BAS and Five-Factor models of personality. Assessment, 16, 71-87. doi:10.1177/1073191108322207.

Ruiz-Padial, E., Mata, J. L., Rodríguez, S., Fernández, M. C. \& Vila, J. (2005). Nonconscious modulation of cardiac defense by masked phobic pictures. International Journal of Psychophysiology, 56, 271-281.

Sánchez, M., Ruiz-Padial, E., Pérez, N., Fernández, M. C., Cobos, P. \& Vila, J. (2002). Modulación emocional de los reflejos defensivos mediante visualización de imágenes afectivas. Psicothema, 14, 702-707.

Vaidyanathan, U., Hall, J. R., Patrick, C. J. \& Bernat, E. M. (2011). Clarifying the role of defensive reactivity deficits in psychopathy and antisocial personality using startle reflex methodology. Journal of Abnormal Psychology, 120, 253-258. [PubMed: 20973594].

Vanman, E. J., Mejia, V. Y., Dawson, M. E., Schell, A. M. \& Raine, A. (2003). Modification of the startle reflex in a community sample: Do one or two dimensions of psychopathy underlie emotional processing? Personality and Individual Differences, 35, 2007-2021. doi: 10.1016/ S0191-8869(03)00052-7.

Vila, J. \& Fernández, M. C. (1989). The cardiac defense response in humans: Effects of predictability and adaptation period. Journal of Psychophysiology, 3, 245-258.

Vila, J., Guerra, P., Muñoz, M. A., Vico, C., Viedma-del Jesús, M. I., Delgado, L. C., Perakakis, P., Kley, E., Mata, J. L. \& Rodriguez, S. (2007). Cardiac defense: From attention to action. International Journal of Psychophysiology, 66, 169-182. doi:10.1016/j.jpsycho.2007.07.004. 